University of New Hampshire

University of New Hampshire Scholars' Repository

Physics Scholarship

Physics

$10-27-2014$

\title{
Fate of the Bose insulator in the limit of strong localization and low Cooper-pair density in ultrathin films
}

Shawna M. Hollen

University of New Hampshire - Main Campus

G. E. Fernandes

Brown University

J. M. Xu

Brown University

J M. Valles Jr.

Brown University

Follow this and additional works at: https://scholars.unh.edu/physics_facpub

Part of the Condensed Matter Physics Commons

\section{Recommended Citation}

S. Hollen, G. E. Fernandes, J. M. Xu, and J. M. Valles, Jr., 'Fate of the Bose insulator in the limit of strong localization and low Cooper-pair density in ultrathin films', Physical Review B, vol. 90, no. 14, pp.

140506-1 - 140506-5, Oct. 2014.

This Article is brought to you for free and open access by the Physics at University of New Hampshire Scholars' Repository. It has been accepted for inclusion in Physics Scholarship by an authorized administrator of University of New Hampshire Scholars' Repository. For more information, please contact Scholarly.Communication@unh.edu. 


\title{
Fate of the Bose insulator in the limit of strong localization and low Cooper-pair density in ultrathin films
}

\author{
S. M. Hollen, ${ }^{1, *}$ G. E. Fernandes, ${ }^{2, \dagger}$ J. M. Xu,${ }^{1,2, \ddagger}$ and J. M. Valles, Jr. ${ }^{1}$ \\ ${ }^{1}$ Department of Physics, Brown University, Providence, Rhode Island 02912, USA \\ ${ }^{2}$ School of Engineering, Brown University, Providence, Rhode Island 02912, USA \\ (Received 29 April 2014; revised manuscript received 6 October 2014; published 27 October 2014)
}

\begin{abstract}
A Bose insulator composed of a low density of strongly localized Cooper pairs develops at the two-dimensional superconductor to insulator transition (SIT) in a number of thin film systems. Investigations of ultrathin amorphous $\mathrm{PbBi}$ films far from the SIT described here provide evidence that the Bose insulator gives way to a second insulating phase with decreasing film thickness. At a critical film thickness $d_{c}$ the magnetoresistance changes sign from positive, as expected for boson transport, to negative, as expected for fermion transport, signs of local Cooper-pair phase coherence effects on transport vanish, and the transport activation energy exhibits a kink. Below $d_{c}$ pairing fluctuation effects remain visible in the high-temperature transport while the activation energy continues to rise. These features show that Cooper pairing persists and suggest that the localized unpaired electron states involved in transport are interspersed among regions of strongly localized Cooper pairs in this strongly localized, low Cooper-pair density phase.
\end{abstract}

DOI: 10.1103/PhysRevB.90.140506

PACS number(s): 74.81.-g, 73.50.-h, 74.25.F-, 74.40.Kb

Particle exchange statistics influence a range of many body phenomena in traditional condensed matter and cold atom systems. Localization transitions and transport in quantum matter, for example, depend on whether fermions or bosons are involved [1-9]. Noninteracting fermions can undergo a disorder induced conductor to insulator transition while bosons cannot. Bosons require repulsive interactions to form the conducting state [10]. Otherwise, without the Pauli principle, they all condense into the lowest energy single particle state, which is always localized in a disordered potential [1,2]. Effects of particle statistics also appear in transport when carrier hopping between localized states dominates [3]. The individual hopping transitions often involve virtual transitions to nearby occupied states. Because these indirect transitions involve particle exchange, their contribution to the total hopping transition probability depends on whether the carriers are fermions or bosons. This dependence has a strong influence on the magnetoresistance (MR), making it positive for bosons $[4,11]$ and negative for fermions $[5,6]$.

Thin film superconductors near the two-dimensional (2D) quantum superconductor to insulator transition (SIT) provide a unique arena for observing quantum statistics effects in many body systems [7,12]. Researchers now reproducibly create high sheet resistance films $\left(R_{N} \approx h / 4 e^{2}\right)$ that can undergo either a superconductor to Fermi insulator (FI) $[7,13,14]$ or superconductor to Bose insulator (BI) [15-19] transition. The FIs exhibit transport characteristics consistent with the incipient $T=0$ Anderson localization of unpaired, noninteracting electrons. All signs of the phase coherent condensate of the superconductor disappear at a critical thickness [14]. Deep in the FI phase, the MR is negative [20]. The BIs, by contrast, exhibit activated transport $[19,21]$, which is consistent with

\footnotetext{
*shawna_hollen@brown.edu; current address: Center for Emergent Materials, The Ohio State University, Columbus, Ohio 43210, USA. †gustavo_fernandes@brown.edu

†jimmy_xu@brown.edu

§ames_valles_jr@brown.edu
}

a repulsive interaction induced Mott insulator phase. Their giant positive magnetoresistance is consistent with incoherent tunneling of bosons [4]. The existence of the bosons in the form of Cooper pairs (CPs) has been established by signs of local phase coherence among particles having charge $2 e[22,23]$ and tunneling experiments showing an energy gap in the density of states [24]. This Bose insulator has drawn general interest because of its proximity to a quantum critical point and its similarities to the nonsuperconducting pseudogapped state in high $T_{c}$ superconductors [25].

Experimental results presented here probe films far from the SIT to determine the fate of the Bose insulator phase. Scenarios for this regime, where localization effects are strongest and the Cooper-pair density is lowest, diverge on whether or not localized pairs survive [26,27]. One might expect fermionic characteristics to emerge as CPs break into their constituents. A strongly disordered single particle potential can break pairs due to a reduced density of states and stronger repulsive interactions [28]. Even a periodic potential of sufficient amplitude can depair a BCS state to produce a Mott insulator, as discussed for atomic Fermi systems [8]. The reduction in the localization volume due to the potential can also make the energy level spacing large enough to be unfavorable for pair formation [29]. Alternatively, localization can enhance pair binding interactions by increasing wave function overlap [30,31]. These effects can occur when the pair spacing becomes comparable to or greater than the pair size. We examined this regime using ultrathin nanopatterned amorphous $\mathrm{Pb}_{0.9} \mathrm{Bi}_{0.1}(a-\mathrm{PbBi})$ films (see below) [32]. Their magnetoresistance characteristics indicated that the Bose insulator's thermally activated boson transport gives way to thermally activated fermion transport below a well defined thickness. High-temperature signatures of pairing fluctuations weaken but persist with decreasing thickness. The activation energy continues to rise, which is consistent with increasing single electron localization. Thus, pair unbinding turns on in the strong localization regime so that the thinnest films appear to support a mixture of fermions and remanent localized bosons. We anticipate these findings will inform attempts to describe the transport observed in many 

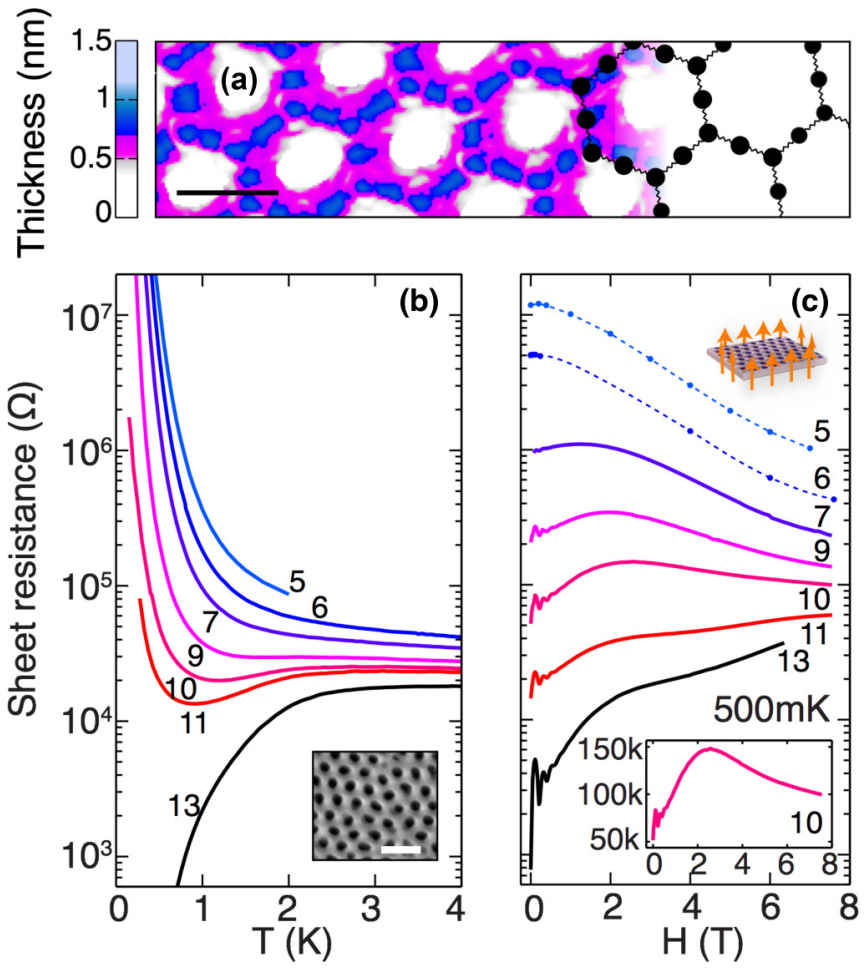

FIG. 1. (Color online) (a) Structure of the NHC films as determined by a combination of atomic force micrographs and transport data [15]. Blue regions are thick enough to support Cooper-pair formation, and pink regions are not. Superconducting islands (dots) are connected by weak links (resistor symbols) and form an organized network. The scale bar is $100 \mathrm{~nm}$. (b) Sheet resistance as a function of temperature for a series of $a$-PbBi NHC films of increasing film thickness. Film thicknesses from top to bottom are 0.350, 0.360, $0.370,0.385,0.395,0.400$, and $0.413 \mathrm{~nm}$. Inset: Scanning electron micrograph of AAO substrate. The scale bar is $200 \mathrm{~nm}$. (c) Sheet resistance as a function of perpendicular magnetic field (as in upper inset) for the films in (b) at $500 \mathrm{mK}$ on a $\log R$ scale. Film 10 is shown on a linear scale in the lower inset.

thin film Bose insulator systems [19,21] while encouraging analogies to experiments on cold fermion atoms with attractive interactions in optical lattices [8,33-36].

The structure of the films investigated [15] is well suited for studying fermion pairing in the localized regime. This special morphology is imposed by the anodized aluminum oxide (AAO) substrates [37]. The films are formed by quench depositing a metal, in this case [32] $\mathrm{Pb}_{0.9} \mathrm{Bi}_{0.1}$, onto a quench deposited $1 \mathrm{~nm}$ thick layer of $\mathrm{Sb}$ onto an AAO substrate held at $8 \mathrm{~K}$ on the mixing chamber of a dilution refrigerator (see Ref. [15] for a more detailed description). The AAO substrate surface has a triangular array of holes with each hole encircled by a sixfold pattern of surface height undulations. The combination of quench condensation and the substrate surface pattern produces hexagonally patterned nanohoneycomb (NHC) amorphous films with 12 thickness undulations around each hole [15], as shown in Fig. 1(a). Because $T_{c}$ of $a$-PbBi depends on film thickness, these undulations form superconducting dots connected by thinner film weak links in an organized network near the SIT [Fig. 1(a), right]. Previous experiments using flat Si substrates with holes, which produced only Fermi insulator behavior, established that these surface undulations are essential for inducing Bose insulator behavior [14,38]. With increasing film thickness, the growth of the weak link coupling leads to the insulator to superconductor transition [Fig. 1(b)] at a critical thickness $d_{\mathrm{SIT}}$ at which the weak link resistance is close to the quantum of resistance for Cooper pairs [15]. Moreover, the multiply connected geometry imposed by the hole array enables a proxy for the existence of the Cooper pairs. In a perpendicular magnetic field [see Fig. 1(c), upper inset], quantum interference among Cooper pairs give rise to MR oscillations with a period $\mu_{0} H_{\mathrm{M}}=\phi_{0} n_{\text {holes }}$, where $n_{\text {holes }}$ is the areal density of the hole array. This effect revealed Cooper pairs in insulating films to establish the Bose insulator phase [22].

An estimate (see the Supplemental Material [39]) shows that the average number of CPs per dot in these films is less than one near the SIT, which makes their survival uncertain in thinner, more insulating films. In the estimate, $\left\langle N_{\mathrm{CP}, \mathrm{dot}}\right\rangle=$ $\frac{1.7}{8} \frac{k_{B} T_{c}}{\delta}=\frac{1.7}{8} N\left(E_{F}\right) V_{\mathrm{dot}} k_{B} T_{c}$, where $\delta$ is the energy level spacing on an isolated dot, $T_{c}$ is the pairing energy scale, $V_{\text {dot }}$ is the dot volume, and $N\left(E_{F}\right)$ is the density of states at the Fermi energy. Using the known film geometry, $\mathrm{Pb}$ 's $N\left(E_{F}\right)$ and bulk $T_{c}$ yields $\left\langle N_{\mathrm{CP} \text {,dot }}\right\rangle \approx 0.2$. Thus, in a BCS picture the energy level spacing is likely to become too large as the dots uncouple for pairing to be favorable. On the other hand, this estimate implies that the pair spacing exceeds the coherence length $\approx 15 \mathrm{~nm}$ [40], which drives the system into the strongly coupled BEC-like regime where pairing interactions are expected to increase with decreasing interdot coupling [30,31].

We drove the system into the strongly localized regime by limiting the evaporated film thickness and then performed magnetoresistance measurements to examine the effects. The simultaneous evolution of the resistance as a function of temperature $R(T)$ and the MR is displayed in Figs. 1(b) and 1(c). Superconducting film 13 shows a strong positive MR with superimposed low-field period $2 e$ MR oscillations [Fig. 1(c)] due to quantum interference of CPs in loops defined by the holes in the films. These oscillations and the positive MR persist into the insulating state. Insulating films nearest the SIT (9-11) show oscillations and a positive MR that peaks at $\sim 2-3 \mathrm{~T}$. This large MR peak, shown on a linear scale for film 10 [Fig. 1(b), lower inset], is considered the signature of the Cooper-pair insulator (CPI) phase. With decreasing film thickness, the positive slope of the low-field MR and the oscillation amplitude decrease together. Film 7 shows hints of both while film 6 , which is thinner, lacks oscillations and exhibits a negative MR [Figs. 1(c) and 2(a)]. To bring out this change in MR, we define the oscillation amplitude,

$$
A=\ln R_{H_{\mathrm{M}} / 2}-\left(\ln R_{0}+\ln R_{H_{\mathrm{M}}}\right) / 2,
$$

and the slope,

$$
m=\ln R_{H_{\mathrm{M}}}-\ln R_{0},
$$

and depict them in Fig. 2(a). Both $A$ and $m$, measured at $500 \mathrm{mK}$, diminish with decreasing film thickness (increasing disorder) [Figs. 2(b) and 2(c)] and vanish together at a critical thickness $d_{c}<d_{\text {SIT }}$ [Fig. 2(d)].

Throughout the insulator phase the $R(T)$ fit to a simply activated form, $R=R_{0} e^{T_{0} / T}$, at low fields (below $\sim 4 \mathrm{~T}$ ), as in Fig. 3(a). The activation energy depends on magnetic field, 

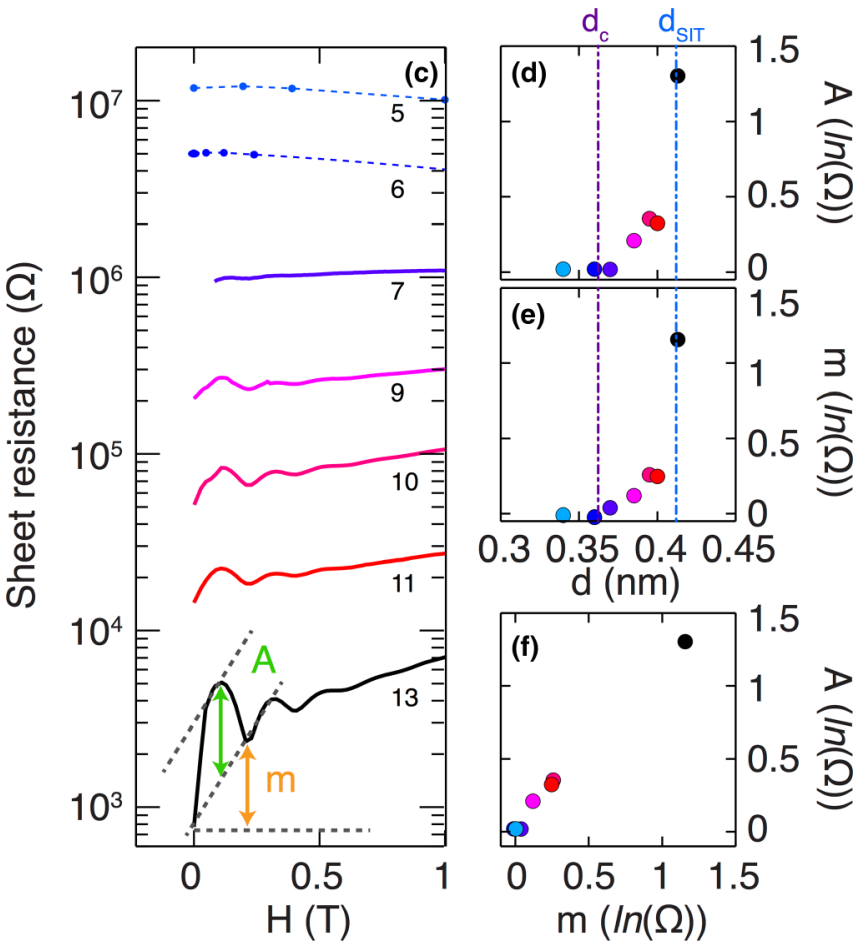

FIG. 2. (Color online) (a) Sheet resistance as a function of perpendicular magnetic field for the same data as in Fig. 1(c) for a limited range to show the low-field region. The definitions of oscillation amplitude $(A)$ and slope $(m)$ are illustrated for film 13 (black). (b) Amplitude of the first magneto-oscillation in the resistance as a function of film thickness. (c) Low-field slope of the MR as a function of film thickness. (d) Oscillation amplitude vs slope to show the simultaneous disappearance of the two.

oscillating and rising as the MR, as previously shown [38]. The oscillations and rise in $T_{0}(H)$ also appear to go to zero at $d_{c}$ as $A$ and $m$ (see Fig. 1 in the Supplemental Material). We define $d_{c}=0.36 \mathrm{~nm}$ and $d_{\text {SIT }}=0.41 \mathrm{~nm}$ [in Fig. 2(d) and Figs. 4(c) and 4(d)] using these temperature-independent data parameters. Significantly, the thickness dependences of the zero-field transport parameters $T_{0}$ and $R_{0}$, shown in Figs. 3(b) and 3(c), appear to change abruptly at the critical film thickness where the magnetotransport changes. Previously, we reported a nearly linear dependence of $T_{0}(H=0 \mathrm{~T})$ on thickness and a featureless, monotonic $R_{0}(d)$. By extending our measurements to thinner films and a finer selection of film thicknesses, a distinct shift in $T_{0}(d)$ and a coincident jump in $R_{0}(d)$ at $d=d_{\mathrm{c}}$ became apparent [Figs. 3(b) and 3(c)]. Upon reexamination of previous experiments on NHC films, we find similar features in the thickness dependence of the activation energy and prefactor at high disorder that also correlate with the appearance of MR oscillations (see Fig. 2 in the Supplemental Material).

The appearance of negative MR, which is strong evidence that fermions (i.e., electrons) become the charge carriers, begs the question of whether CPs exist below $d_{c}$. It is interesting to address this question as this sign change may be a universal feature in the strong disorder limit since it appears in other systems, such as indium oxide films [26,41]. We can probe for the survival of CPs by examining the $R(T)$. With decreasing $T$, insulating films near the SIT exhibit a strong reentrant dip before the onset of a simply activated rise [see films 9, 10, and 11 in Fig. 1 and films 8-12 in Fig. 3(a)]. The dip signals the formation of $\mathrm{CP}$ islands, which effectively shunt more resistive regions of a film [42]. To track this pairing feature into the strongly insulating regime where the activated behavior tends to mask it, we examine the derivative

$$
T_{0, \text { local }}(1 / T)=\frac{d \ln (R)}{d(1 / T)}
$$

of the Arrhenius curves in Fig. 3(a). It asymptotes to a constant value at low temperatures that matches the activation energies found by fitting the Arrhenius curves. The onset of reentrant behavior appears as an inflection point in $R(T)$ and a minimum in $T_{0, \text { local }}$. What is particularly striking is that the reentrant feature is present for every film in the series, including those with $d<d_{c}$ and $T_{0}$ 's as high as $5 \mathrm{~K}$.

Corroborating evidence for remanent pairing appears through comparing the $R(T)$ of NHC films with those of simultaneously grown unpatterned reference films. The uniform thickness of a reference film corresponds to the maximum thickness regions in a neighboring NHC film [15]. Figure 4 makes this comparison with data from a separate experiment that yielded similar, but less detailed, results on the NHC films to those presented in Figs. 1-3. While all of the NHC films are insulators over a thickness range spanning $d_{c}$ [Fig. 4(a)], their corresponding reference films are superconductors [Fig. 4(b)]. These data affirm that all insulating NHC films examined here have regions that are thick enough to support pairing interactions.

Recent theories that consider quantum interference effects on CP transport suggest why the fates of the MR oscillations and the positive MR intertwine. Here, we highlight two that emerge from distinct physical models of the Cooper-pair insulator phase that are most applicable to the structure apparent in the NHC films: Josephson junction array (JJA) [21,43-45] and boson hopping interference (BHI) $[4,6,11,46,47]$. In JJA models, films consist of superconducting islands coupled to their neighbors by tunnel junctions. The interisland tunneling rates depend on the difference between the order parameter phases on neighboring islands, which makes them susceptible to magnetic fields. Consequently, the average tunneling rate oscillates in transverse fields with a period corresponding to one flux quantum per unit cell of the array [48], as observed here. The accompanying positive MR can be attributed to the magnetic field reducing $E_{J}$ by inducing quantum interference within the finite width tunnel junctions [48]. The NHC film link dimensions are large enough $(>20 \mathrm{~nm})$ that a $\sim 2 \mathrm{~T}$ magnetic field would be sufficient to cause considerable interference. In BHI models, CP hopping between localized states dominates transport [4]. The hopping probability depends on the superposition of amplitudes of virtual hopping trajectories that involve intermediate states. In zero magnetic field, boson trajectories interfere only constructively [4]. Applied magnetic flux through the areas defined by the trajectories destroys the perfect constructive interference to produce positive MR. For well defined loops as in an array, the MR is expected to oscillate [49]. The positive MR can be attributed to interference effects within the links in the arrays. Thus, both models indicate that quantum interference effects that are readily apparent in the MR oscillations make the MR positive for boson transport. 

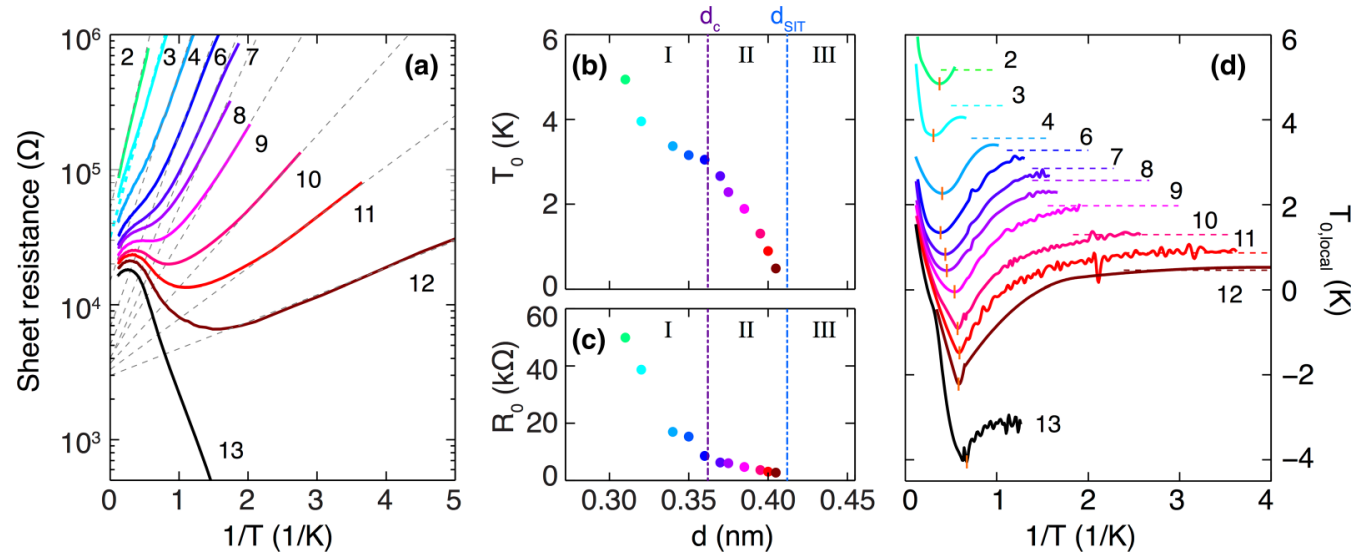

FIG. 3. (Color online) (a) Sheet resistance vs inverse temperature for all films measured, omitting film 5 for lack of data above $1 \mathrm{~K}$. Dashed lines are Arrhenius fits to $R=R_{0} e^{T_{0} / T}$ showing simply activated behavior. (b) Zero-field activation energies $\left(T_{0}\right)$ and (d) prefactors $\left(R_{0}\right)$ obtained from fits to Arrhenius curves shown in (a) vs film thickness. The uncertainty in the fit parameters is comparable to the size of the data points. The vertical dashed lines mark $d_{c}$ and $d_{\text {SIT }}$ as defined in the text. (d) Differentiated curves in (a), $T_{0, \text { local }}$ [defined by Eq. (3)], vs inverse temperature. Signs of reentrance are clear for each film. The vertical orange bar marks the position of the inflection point in $R(T)$ for each film. $T_{0}$ from low-temperature Arrhenius fits are shown as horizontal dashed lines.

Possible explanations for the fermion transport signal emerging at $d<d_{c}$ come from two prevalent models [44,50] of the giant magnetoresistance peak exhibited by the Cooper-pair insulator [18,19,38] (cf. Fig. 1). Gantmakher [44] proposed that quasiparticle tunneling between superconducting islands dominates on the negative magnetoresistance side of the peak. $\Delta$, the activation energy for the process, decreases with magnetic field to produce the negative MR. The crossover from boson to fermion dominated transport occurs when the magnetic field reduces the pair binding energy $2 \Delta$ below the Coulomb blockade energy for pair transport $E_{C}$ as in Josephson junction array models [51]. If this quasiparticle tunneling takes over in the thinnest films at zero magnetic field, then the observed rise in the activation energy with decreasing thickness [Fig. 3(b)] implies that $\Delta$ increases with localization. This increase would be unexpected within a BCS pairing model [52], though it has been proposed for strongly localized states in low Cooper-pair density systems $[31,53]$. An alternative process comes up if a
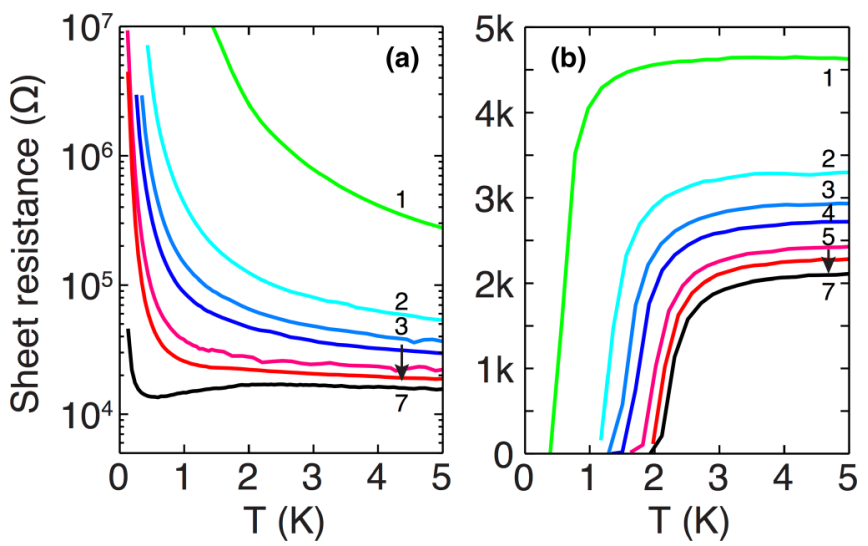

FIG. 4. (Color online) Sheet resistance vs temperature for (a) a series of insulating $a$-PbBi NHC films approaching the SIT, and (b) the corresponding superconducting reference films grown simultaneously on a neighboring polished glass substrate. Film thicknesses from top to bottom are approximately $0.42,0.47,0.49$, $0.51,0.54,0.55$, and $0.56 \mathrm{~nm}$. fraction of the dots become too small and weakly coupled to their neighbors to support pairing. Electrons traversing this mixture of localized boson and fermion regions would have to overcome a Coulomb blockade to enter neighboring paired dots. As described by Dubi, Meir, and Avishai [50], this barrier grows as the decreasing film thickness causes the paired dots to shrink. This effect causes the activation energy to rise with decreasing film thickness, as qualitatively observed. In a magnetic field, paired dots become normal to reduce the barrier to electron transport and render the MR negative. This Bose-fermion mixture explanation seems more likely as it is consistent with the reduction in the pairing interaction in reference films with decreasing thickness (cf. Fig. 4).

The microscopic conditions necessary for this fermionic transport regime to develop remain to be defined. The spatial periodicity of the thickness variations in the NHC films that creates dots with very low Cooper-pair occupancy makes them appear amenable to analysis by Hubbard models applied to cold fermionic atoms in optical lattices $[8,9,33,35,54]$. The dots become the lattice sites and the weak links the intersite tunneling channels. Experimental observations of superfluid to insulator transitions $[9,33]$ controlled by lattice depth and/or the interaction potential that are similar to the SIT in films add weight to this analogy. Theories have predicted the existence of at least two different insulating phases that can develop, a Mott and a band insulator $[8,35,54]$. These findings raise the possibility that a pair insulator can cross over to an electron insulator if the pairing interaction changes in the insulating phase [54]. Additional theoretical studies using parameters relevant to NHC films may be able to explain the well defined character of this transition and the behavior of the microscopic energy scales governing the transport in NHC films.

We have reported data that show a transition from bosonic to fermionic carrier statistics deep in the insulating phase of $a$ - $\mathrm{PbBi}$ NHC films. Moreover, the evidence supports that this transition signals entry into a phase consisting of a mixture of bosons and fermions. Experiments on unpatterned homogeneous thin film systems, such as those revealing a sign change in MR with increased disorder [26] or electrostatic 
doping [41], suggest that this mixed particle statistics regime likely develops far from the SIT in general.

We benefited from discussions with Markus Mueller, Nandini Trivedi, Mason Swanson, and Danny Shahar. We are also glad to acknowledge James Joy for his assistance in the data collection. We are grateful for the support of the NSF through Grants No. DMR-1307290 and No. DMR-0907357, and by the AFRL and the AFOSR.
[1] M. P. A. Fisher, P. B. Weichman, G. Grinstein, and D. S. Fisher, Phys. Rev. B 40, 546 (1989).

[2] X. Yu and M. Mueller, Ann. Phys. 337, 55 (2013).

[3] V. L. Nguyen, B. Z. Spivak, and B. I. Shklovskii, JETP Lett. 43, 44 (1986).

[4] M. Mueller, Europhys. Lett. 102, 67008 (2013).

[5] V. L. Nguyen, B. Z. Spivak, and B. I. Shklovskii, JETP Lett. 41, 42 (1985).

[6] H. L. Zhao, B. Z. Spivak, M. P. Gelfand, and S. C. Feng, Phys. Rev. B 44, 10760 (1991).

[7] D. B. Haviland, Y. Liu, and A. M. Goldman, Phys. Rev. Lett. 62, 2180 (1989).

[8] V. A. Kashurnikov and A. V. Krasavin, J. Exp. Theor. Phys. 111, 180 (2010).

[9] T. Esslinger, Annu. Rev. Condens. Matter Phys. 1, 129 (2010).

[10] M. Wallin, E. S. Sorensen, S. M. Girvin, and A. P. Young, Phys. Rev. B 49, 12115 (1994).

[11] S. V. Syzranov, A. Moor, and K. B. Efetov, Phys. Rev. Lett. 108, 256601 (2012).

[12] V. Dobrosavljevic, N. Trivedi, and J. M. Valles, Jr., ConductorInsulator Quantum Phase Transitions (Oxford University Press, Oxford, UK, 2012).

[13] J. M. Valles, Jr., R. C. Dynes, and J. P. Garno, Phys. Rev. Lett. 69, 3567 (1992)

[14] S. M. Hollen, G. E. Fernandes, J. M. Xu, and J. M. Valles, Jr., Phys. Rev. B 87, 054512 (2013).

[15] S. M. Hollen, H. Q. Nguyen, E. Rudisaile, M. D. Stewart, Jr., J. Shainline, J. M. Xu, and J. M. Valles, Jr., Phys. Rev. B 84, 064528 (2011).

[16] B. Sacépé, C. Chapelier, T. I. Baturina, V. M. Vinokur, M. R. Baklanov, and M. Sanquer, Phys. Rev. Lett. 101, 157006 (2008).

[17] M. A. Steiner, N. P. Breznay, and A. Kapitulnik, Phys. Rev. B 77, 212501 (2008).

[18] T. I. Baturina, A. Y. Mironov, V. M. Vinokur, M. R. Baklanov, and C. Strunk, Phys. Rev. Lett. 99, 257003 (2007).

[19] G. Sambandamurthy, L. W. Engel, A. Johansson, and D. Shahar, Phys. Rev. Lett. 92, 107005 (2004).

[20] N. Markovic, A. M. Mack, G. Martinez-Arizala, C. Christiansen, and A. M. Goldman, Phys. Rev. Lett. 81, 701 (1998).

[21] M. V. Fistul, V. M. Vinokur, and T. I. Baturina, Phys. Rev. Lett. 100, 086805 (2008).

[22] M. D. Stewart, A. Yin, J. M. Xu, and J. M. Valles, Jr., Science 318, 1273 (2007).

[23] G. Kopnov, O. Cohen, M. Ovadia, K. H. Lee, C. C. Wong, and D. Shahar, Phys. Rev. Lett. 109, 167002 (2012).

[24] D. Sherman, G. Kopnov, D. Shahar, and A. Frydman, Phys. Rev. Lett. 108, 177006 (2012).

[25] B. Sacépé, C. Chapelier, T. I. Baturina, V. M. Vinokur, M. R. Baklanov, and M. Sanquer, Nat. Commun. 1, 140 (2010).

[26] D. Shahar and Z. Ovadyahu, Phys. Rev. B 46, 10917 (1992).

[27] V. F. Gantmakher and V. T. Dolgopolov, Phys.-Usp. 53, 1 (2010).

[28] D. Belitz and T. R. Kirkpatrick, Rev. Mod. Phys. 66, 261 (1994).
[29] P. Anderson, J. Phys. Chem. Solids 11, 26 (1959).

[30] M. V. Feigel'man, L. B. Ioffe, V. E. Kravtsov, and E. Cuevas, Ann. Phys. 325, 1390 (2010).

[31] K. Bouadim, Y. L. Loh, M. Randeria, and N. Trivedi, Nat. Phys. 7, 884 (2011)

[32] S. M. Hollen, J. Shainline, J. M. Xu, and J. M. Valles, Jr., Physica C 486, 23 (2013)

[33] J. K. Chin, D. E. Miller, Y. Liu, C. Stan, W. Setiawan, C. Sanner, K. Xu, and W. Ketterle, Nature (London) 443, 961 (2006).

[34] S. Datta, V. N. Singh, and P. Majumdar, Phys. Rev. A 89, 053609 (2014).

[35] Z. Shen, L. Radzihovsky, and V. Gurarie, Phys. Rev. Lett. 109, 245302 (2012).

[36] R. Watanabe and M. Imada, Phys. Rev. A 80, 043624 (2009).

[37] A. J. Yin, J. Li, W. Jian, A. J. Bennett, and J. M. Xu, Appl. Phys. Lett. 79, 1039 (2001).

[38] H. Q. Nguyen, S. M. Hollen, M. D. Stewart, Jr., J. Shainline, A. Yin, J. M. Xu, and J. M. Valles, Jr., Phys. Rev. Lett. 103, 157001 (2009).

[39] See Supplemental Material at http://link.aps.org/supplemental/ 10.1103/PhysRevB.90.140506 for disappearance of oscillations in the field dependence of the activation energy, the consistent observation of a kink in the thickness dependence of the activation energy over many NHC film experiments, and an estimation of the number of CPs per dot

[40] J. A. Chervenak and J. M. Valles, Jr., Phys. Rev. B 54, R15649(R) (1996).

[41] Y. Lee, A. Frydman, T. Chen, B. Skinner, and A. M. Goldman, Phys. Rev. B 88, 024509 (2013).

[42] H. M. Jaeger, D. B. Haviland, B. G. Orr, and A. M. Goldman, Phys. Rev. B 40, 182 (1989).

[43] M. Tinkham, D. W. Abraham, and C. J. Lobb, Phys. Rev. B 28 , 6578 (1983).

[44] V. F. Gantmakher, M. V. Golubkov, V. T. Dolgopolov, G. E. Tsydynzhapov, and A. A. Shashkin, JETP Lett. 68, 363 (1998).

[45] R. P. Barber, Jr., S.-Y. Hsu, J. M. Valles, Jr., R. C. Dynes, and R. E. Glover, III, Phys. Rev. B 73, 134516 (2006).

[46] M. Müller, Ann. Phys. 18, 849 (2009).

[47] A. Gangopadhyay, V. Galitski, and M. Muller, Phys. Rev. Lett. 111, 026801 (2013).

[48] M. G. Forrester, H. J. Lee, M. Tinkham, and C. J. Lobb, Phys. Rev. B 37, 5966(R) (1988).

[49] M. Müller (private communication).

[50] Y. Dubi, Y. Meir, and Y. Avishai, Phys. Rev. B 73, 054509 (2006).

[51] P. Delsing, C. D. Chen, D. B. Haviland, Y. Harada, and T. Claeson, Phys. Rev. B 50, 3959 (1994).

[52] T. R. Kirkpatrick and D. Belitz, Phys. Rev. Lett. 68, 3232 (1992).

[53] M. V. Feigel'man, L. B. Ioffe, V. E. Kravtsov, and E. A. Yuzbashyan, Phys. Rev. Lett. 98, 027001 (2007).

[54] H. Zhai and T.-L. Ho, Phys. Rev. Lett. 99, 100402 (2007). 